\title{
Annarosa Poli, «Il Journal di Delacroix»
}

\section{Annalisa Bottacin}

\section{OpenEdition}

\section{Journals}

\section{Edizione digitale}

URL: http://journals.openedition.org/studifrancesi/8963

DOI: 10.4000/studifrancesi.8963

ISSN: 2421-5856

\section{Editore}

Rosenberg \& Sellier

\section{Edizione cartacea}

Data di pubblicazione: 1 octobre 2008

Paginazione: 470-471

ISSN: 0039-2944

\section{Notizia bibliografica digitale}

Annalisa Bottacin, «Annarosa Poli, «ll Journal di Delacroix»», Studi Francesi [Online], 155 (LII | II) | 2008 , online dal 30 novembre 2015, consultato il 12 janvier 2021. URL: http://journals.openedition.org/ studifrancesi/8963 ; DOI: https://doi.org/10.4000/studifrancesi.8963

Questo documento è stato generato automaticamente il 12 janvier 2021.

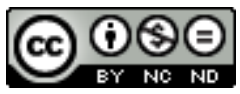

Studi Francesi è distribuita con Licenza Creative Commons Attribuzione - Non commerciale - Non opere derivate 4.0 Internazionale. 


\title{
Annarosa Poli, «Il Journal di Delacroix»
}

\author{
Annalisa Bottacin
}

\section{NOTIZIA}

ANNAROSA POLI, «Il Journal di Delacroix», in Giornate particolari. Diari, memorie e cronache, a cura di Bianca Tarozzi, Verona, Ombre Corte, 2006 («Culture»), pp. 74-91.

1 L'accurata esegesi di Annarosa Poli ripercorre le principali tappe di tre opere scritturali del celebre pittore Eugène Delacroix e precisamente il Journal giovanile, quello della maturità e il Dictionnaire des beaux-arts, rimasto per altro allo stadio di abbozzo, sottolineando come tale produzione vada de pair con l'opera pittorica. Ammirato da Baudelaire, esaltato da Gautier, l'innovatore della pittura francese dell'Ottocento partecipò a vari "Salons" parigini, esponendo quadri che saranno infatti recensiti con dovizia di particolari da Théophile Gautier in varie riviste. Profonda fu anche l'amicizia che legò Delacroix a Stendhal; il grenoblese non manca, nelle lettere inviate dall'Italia agli amici parigini, di ricordare sovente con affetto e profonda stima il Maestro, che a sua volta lo ringrazia, in un biglietto dell'ottobre del 1828, per un articolo firmato dallo stesso Beyle e apparso nella rivista britannica «Athenæum» nel maggio precedente, in cui lo scrittore così si esprime, riferendosi alla tela raffigurante La Mort de Sardanapale, esposta l'anno précédente: «M. Delacroix, jeune peintre d'un talent considérable, a exposé un tableau représentant Sardanapale mourant. Cette œuvre semble avoir été inspirée par le génie de Lord Byron; la même énergie, la même profonde détresse, et, si je puis dire, le même ton satanique animent l'œuvre du peintre et celle du poète» (Stendhal, Chroniques pour l'Angleterre, VII, 275). Come sostiene l'autore del Rouge la fine del re, che decide di morire con tutte le sue concubine, i suoi cavalli e i suoi cani favoriti è qui pretesto per una pittura tumultuosa, fremente e accesissima. Ciò che evidenzia Poli nel suo corposo e appassionante lavoro è dunque la figura di un giovane artista che a ventiquattro anni decide "d'écrire un journal», la cui prima pagina è stilata il 3 settembre 1822, anno in cui Delacroix espone al "Salon" parigino quel Dante 
et Virgile aux enfers, che suscitò scalpore per il soggetto inconsueto e l'intensa drammaticità della composizione, e che farà dire a Baudelaire: "Aucun tableau ne révèle mieux, à mon avis, l'avenir d'un grand peintre que celui de M. Delacroix, représentant Le Dante et Virgile aux enfers. C'est là surtout qu'on peut remarquer ce jet de talent, cet élan de la supériorité naissante...» (Baudelaire, Salon de 1846, in œuvres complètes, Paris, Gallimard, «Bibliothèque de la Pléiade», 1976, t. II, p. 427). L'importanza di essere chiamato al "Salon" è assai rilevante, come ben scrive Poli, "[u]na tale consacrazione... "continua la studiosa» era veramente rara, un onore eccezionale per un giovane pittore» (p. 73), dato che Delacroix era nato nel 1798. Le pagine del Journal sono personalissime, «Delacroix non si rifà a nessun modello [e] a questo punto di partenza corrisponde quello della pratica artistique» (p. 75); almeno all'inizio la scrittura racchiude le sensazioni più vive legate ad ogni nuova esplosione pittorica, i momenti di turbamento, di indecisione, ricalcando alcune frasi di sant'Agostino, «così, nel 1824, il pittore segue nel Diario il lungo procedere del quadro Les Massacres de Scio con i numerosi cambiamenti» (p.77). Il Journal de jeunesse s'interrompe nell'ottobre del 1824: "più il gusto dell'ornamento e della calligrafia seduce l'autore, più il diario si vuota di ogni contenuto e di ogni senso» (p. 78). Pur tuttavia nel 1847 la scrittura viene ripresa, ma «[m]entre Delacroix vedeva nel Diario nel 1822 una guida morale e finanziaria, il nuovo Diario è dedicato ai piaceri della sensazione e della memoria» (p.79). Un'altra opera era comunque nella mente del pittore, un'opera cui teneva particolarmente dato l'alto ruolo d'artista, ma che non vedrà la luce. Si tratta del Dictionnaire des beaux-arts, la cui prima idea risale all'ottobre del 1853. Basandosi sulle ben note Réflexions critiques sur la poésie et la peinture dell'Abbé $\mathrm{Du}$ Bos, Delacroix concepisce un articolo, dal titolo «Autorité», ma, come ben rileva la studiosa, il Dictionnaire viene redatto all'interno del Journal, «nella stessa agenda dalla quale non vuole staccarsi rinviando a volte al primo Diario» (p. 83). George Sand, che gli fu amica e che molto ammirò la sua arte, nel suo romanzo La Daniella, scritto nel $1857 \mathrm{e}$ ispirato al suo soggiorno a Frascati di due anni precedente, s'interroga sull'ispirazione artistica, attraverso un personaggio pittore, un soggetto su cui Delacroix si era a lungo dibattuto. E come sottolinea acutamente Annarosa Poli, tutte le pagine dei Diari di Delacroix sono legate intimamente allo studio e alla ricerca delle tecniche dell'arte, ed è ancora George Sand, che tanto ebbe ad influenzare le sue idee artistiche, che con una semplice frase inquadra perfettamente la posizione di Delacroix nel mondo pittoresque: «Il est dans son art l'innovateur et l'oseur par excellence». Il saggio è altresì corredato da un ricco apparato di note (pp. 89-91). 